\title{
Throughput Optimal Policies for Energy Harvesting Wireless Transmitters with Non-Ideal Circuit Power
}

\author{
Jie $\mathrm{Xu}$ and Rui Zhang
}

\begin{abstract}
Characterizing the fundamental tradeoffs for maximizing energy efficiency (EE) versus spectrum efficiency (SE) is a key problem in wireless communication. In this paper, we address this problem for a point-to-point additive white Gaussian noise (AWGN) channel with the transmitter powered solely via energy harvesting from the environment. In addition, we assume a practical on-off transmitter model with non-ideal circuit power, i.e., when the transmitter is on, its consumed power is the sum of the transmit power and a constant circuit power. Under this setup, we study the optimal transmit power allocation to maximize the average throughput over a finite horizon, subject to the time-varying energy constraint and the non-ideal circuit power consumption. First, we consider the off-line optimization under the assumption that the energy arrival time and amount are $a$ priori known at the transmitter. Although this problem is non-convex due to the non-ideal circuit power, we show an efficient optimal solution that in general corresponds to a twophase transmission: the first phase with an $E E$-maximizing on-off power allocation, and the second phase with a $S E$-maximizing power allocation that is non-decreasing over time, thus revealing an interesting result that both the $\mathrm{EE}$ and $\mathrm{SE}$ optimizations are unified in an energy harvesting communication system. We then extend the optimal off-line algorithm to the case with multiple parallel AWGN channels, based on the principle of nested optimization. Finally, inspired by the off-line optimal solution, we propose a new online algorithm under the practical setup with only the past and present energy state information (ESI) known at the transmitter.
\end{abstract}

Index Terms-Energy harvesting, power control, energy efficiency, spectrum efficiency, circuit power.

\section{INTRODUCTION}

G REEN or energy efficient wireless communication has recently drawn significant attention due to the growing concerns about the operator's cost as well as the global environmental cost of using fossil fuel based energy to power cellular infrastructures [1], [2], [3]. To achieve the optimal energy usage efficiency for cellular networks, various innovative "green" techniques across different layers of communication protocol stacks have been proposed [4], [5]. Among others, how to maximize the bits-per-Joule energy efficiency (EE) for the point-to-point wireless link has received a great deal of interest recently [6], [7], [8].

Besides maximizing EE, another key design objective in wireless communication is to maximize the spectrum efficiency (SE) or the number of transmitted bits-per-second-per-Hz (bps/Hz), due to the explosive growth of wireless devices and

J. Xu is with the Department of Electrical and Computer Engineering, National University of Singapore (e-mail:elexjie@nus.edu.sg).

R. Zhang is with the Department of Electrical and Computer Engineering, National University of Singapore (e-mail: elezhang@nus.edu.sg). He is also with the Institute for Infocomm Research, A*STAR, Singapore.
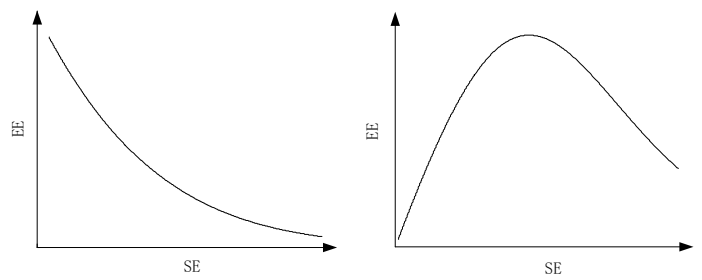

Fig. 1. Tradeoff between EE and SE for the ideal circuit power case of $\alpha=0$ (the left sub-figure) and the non-ideal circuit power case of $\alpha>0$ (the right sub-figure).

applications that require high data rates. In order to design wireless communication systems both energy and spectrum efficiently, the fundamental EE-SE relationship needs to be examined carefully. For the simple additive white Gaussian noise (AWGN) channel with bandwidth $W$ and noise power spectral density $N_{0}$, by applying the Shannon's capacity formula, the $\mathrm{SE}$ and $\mathrm{EE}$ are expressed as $\xi_{\mathrm{SE}}=\log _{2}\left(1+\frac{P}{W N_{0}}\right)$ and $\xi_{\mathrm{EE}}=W \log _{2}\left(1+\frac{P}{W N_{0}}\right) / P$, respectively, with $P$ denoting the transmit power. It thus follows that the optimal EE-SE tradeoff is characterized by $\xi_{\mathrm{EE}}=\frac{\xi_{\mathrm{SE}}}{\left(2^{\xi \mathrm{SE}}-1\right) N_{0}}$, where $\xi_{\mathrm{EE}}$ is a monotonically decreasing function of $\xi_{\mathrm{SE}}$, as shown in the left sub-figure of Fig. 11. In this case, any SE increment will inevitably result in a decrement in EE. However, in practical wireless transmitters, besides the direct transmit power $P$, there also exists non-ideal circuit power consumed when $P>0$, which accounts for the power consumptions at e.g. the AC/DC converter and the analog radio frequency (RF) amplifier, and amounts to a significant part of the total consumed power at the transmitter. Moreover, when there is no data transmission, i.e., $P=0$, the transmitter can turn into a micro-sleep mode [9], by switching off the power amplifier to reduce the circuit power consumption. For the ease of description, in this paper the transmitter status with $P>0$ and $P=0$ are referred to as the on and off modes, respectively. Denote the non-ideal circuit power during an "on" mode as $\alpha \geq 0$ in Watt, the efficiency of the RF chain as $0<\eta \leq 1$, and the power consumed during an "off" mode as $\beta \geq 0$ in Watt. A practical power consumption model for the wireless transmitter is given by [10]

$$
P_{\text {total }}= \begin{cases}\frac{P}{\eta}+\alpha, & P>0 \\ \beta, & P=0,\end{cases}
$$

where $P_{\text {total }}$ denotes the total power consumed at the transmitter. In practice, $\beta$ is generally much smaller as compared to $\alpha$ and thus can be ignored for simplicity [5], [7], [8]. In this paper, we assume $\beta=0$. Therefore, in (1) without loss 
of generality we can further assume $\eta=1$ since $\eta$ is only a scaling constant 1 With the above simplifications, the EE can be re-expressed as $\xi_{\mathrm{EE}}=W \log _{2}\left(1+\frac{P}{W N_{0}}\right) /(P+\alpha)$ for $P>0$ and the resulting new EE-SE tradeoff is shown in the right sub-figure of Fig. 1 for a given $\alpha>0$, from which it is observed that the non-ideal circuit power drastically changes the behavior of the EE-SE tradeoff as compared to the ideal case of $\alpha=0$.

Recently, a new design paradigm for achieving green wireless communication has drawn a great deal of attention, in which wireless terminals are powered primarily or even solely by harvesting the energy from environmental sources such as solar and wind, thereby reducing substantially the energy cost in traditional wireless systems [4], [11]. With the embedded energy harvesting device and rechargeable battery, wireless transmitters can replenish energy from the environment without the need of replacing battery or drawing power from the main grid. Thus, communication utilizing energy harvesting nodes can promisingly achieve a jointly spectrum and energy efficiency maximization goal. However, there are new challenges in designing energy harvesting powered wireless communication, which are not present in traditional systems. For example, the intermittent nature of most practical energy harvesting sources causes random power availability at the transmitter, due to which a new type of transmitter-side power constraint, namely energy harvesting constraint, is imposed, i.e., the energy accumulatively consumed up to any time cannot exceed that accumulatively harvested. As a result, existing EE-SE tradeoffs (cf. Fig. 1) revealed for conventional wireless systems assuming a given constant power supply are not directly applicable to an energy harvesting system, with or without the non-ideal circuit power. It is worth noting that some prior work in the literature has investigated the throughput-optimal power control policies for the energy harvesting wireless transmitter assuming an ideal circuit-power model (i.e., $\alpha=0, \eta=1$, and $\beta=0$ in (1)), in which useful structural properties of the optimal solution were obtained (see e.g. [12], [13], [14] and references therein). However, there is very limited work on studying the effects of the non-ideal circuit power with $\alpha>0$ on the throughput-optimal power allocation for energy harvesting communication systems. To our best knowledge, only [15] has proposed a calculus-based approach to address this problem; however, it does not reveal the structure of the optimal solution. Motivated by the known result that the non-ideal circuit power modifies the EE-SE tradeoff considerably in the conventional case with constant power supply as shown in Fig. 11 we expect that it should also play an important role in the EE-SE tradeoff characterization under the new setup with random power supply due to energy harvesting, which motivates our work.

In this paper, we study the throughput maximization problem for a point-to-point AWGN channel with an energy harvesting powered transmitter over a finite horizon. For the purpose of exposition, we assume that the receiver has a constant power supply (e.g. battery). We also assume that at

${ }^{1}$ Note that the results of this paper can be readily extended to the case with $\eta<1$ by appropriately scaling the obtained solutions. the transmitter, the renewable energy arrives at a discrete set of time instants with variable energy amount. Under this setup, we investigate the effects of the non-ideal circuit power with $\alpha>0$ on the throughput-optimal power allocation as well as the resulting new EE-SE tradeoff. The main contributions of this paper are summarized as follows.

- First, we consider the off-line optimization under the assumption that the energy arrival time and amount for harvesting are a priori known at the transmitter. We show that the optimal power allocation to maximize the average throughput under this setup is a non-convex optimization problem, due to the non-ideal circuit power. Nevertheless, we derive an efficient optimal solution for this problem, which is shown to correspond to a novel two-phase transmission structure: the first phase with an EE-maximizing on-off power allocation, and the second phase with a $S E$-maximizing power allocation that is nondecreasing over time. Thus, we reveal an interesting result that both the EE and SE optimizations are unified in an energy harvesting powered wireless system.

- We then extend the optimal off-line policy for the singlechannel case to the general case with multiple parallel AWGN channels, subject to a total energy harvesting power constraint. Using tools from nested optimization, we transform this problem with multi-dimensional (vector) power optimization to an equivalent one with only one-dimensional (scalar) power optimization, which can then be efficiently solved by the algorithm derived for the single-channel case.

- Furthermore, inspired by the off-line optimal solution, we propose a heuristic online algorithm under the practical setup where only the causal (past and present) energy state information (ESI) for harvesting is assumed to be known at the transmitter. It is shown by simulations that the proposed online algorithm achieves a small performance gap from the throughput upper bound by the optimal off-line solution, and also outperforms other heuristically designed online algorithms.

The rest of this paper is organized as follows. Section III introduces the system model and presents the problem formulation. Section [II] derives the optimal off-line power allocation policy for the single-channel case. Section IV extends the result to the multi-channel case based on the nested optimization. Section $\mathrm{V}$ presents the proposed online algorithm and Section VI evaluates its throughput performance by simulations. Finally, Section VII concludes the paper.

\section{System Model And Problem Formulation}

In this paper, we consider the point-to-point transmission over an AWGN channel with constant channel and coherent detection at the receiver. The transmitter is assumed to replenish energy from an energy harvesting device that collects energy over time from a renewable source (e.g. solar or wind). We consider the block-based energy scheduling with each block spanning over $T$ seconds (secs). We assume that the renewable energy arrives during each block at $N-1$ time instants given by $0<t_{1}<\cdots<t_{N-1}<T$, and the 
energy values collected at these time instants are denoted by $E_{1}, \ldots, E_{N-1}$, respectively. In general, $N \geq 1, t_{i}$, and $E_{i}>0, i=1, \ldots, N-1$, are modeled by an appropriate random process for the given energy source. For convenience, we assume $t_{0}=0$ and denote $E_{0}$ as the initial energy stored in the energy storage device at the beginning time of each block. For the purpose of exposition, we assume that the energy storage device has an infinite capacity in this paper. Moreover, we refer to the time interval between two consecutive energy arrivals as an epoch, and denote the length of the $i$ th epoch as $L_{i}=t_{i}-t_{i-1}, i=1, \ldots, N$; for convenience, we denote $t_{N}=T$.

Suppose that the transmit power over time in each block is denoted by $P(t) \geq 0, t \in(0, T]$. Assume that the maximum transmission rate that can be reliably decoded at the receiver at any time $t$ is a function of $P(t)$, given by $C(t)=R(P(t))$, which satisfies the following properties:

1) $R(P(t)) \geq 0, \forall P(t) \geq 0$, and $R(0)=0$;

2) $R(P(t))$ is a strictly concave function over $P(t) \geq 0$;

3) $R(P(t))$ is a monotonically increasing function over $P(t) \geq 0$.

For example, if adaptive modulation and coding (AMC) is applied at the transmitter, then the achievable rate $C(t)$ is denoted by [16]

$$
R(P(t))=W \log _{2}\left(1+\frac{h P(t)}{\Gamma W N_{0}}\right)
$$

in bits-per-sec (bps), where $\Gamma$ accounts for the gap from the channel capacity due to a practical coding and modulation scheme used; $h>0$ denotes the constant channel power gain.

As discussed in Section \, we assume an on-off transmitter power model given in (1) with $\beta=0$ and $\eta=1$; thus, we rewrite (1) as

$$
P_{\text {total }}(t)=\left\{\begin{array}{cc}
P(t)+\alpha, & P(t)>0 \\
0, & P(t)=0 .
\end{array}\right.
$$

Since the accumulatively consumed energy up to any time at the transmitter cannot exceed the energy accumulatively harvested, the energy harvesting constraint on the total consumed power is given by

$$
\int_{0}^{t_{i}} P_{\text {total }}(t) \mathrm{d} t \leq \sum_{j=0}^{i-1} E_{j}, i=1, \ldots, N .
$$

Thus, the throughput maximization problem over a finite horizon $T$ can be formulated as follows.

$$
\begin{aligned}
\max _{P(t) \geq 0} & \int_{0}^{T} R(P(t)) \mathrm{d} t \\
\text { s.t. } & \int_{0}^{t_{i}} P_{\text {total }}(t) \mathrm{d} t \leq \sum_{j=0}^{i-1} E_{j}, i=1, \ldots, N .
\end{aligned}
$$

The optimal online solution for the above problem with the causal ESI, i.e., for any given $t$, only $E_{i}$ 's with $t_{i} \leq t$ are known at the transmitter, can be numerically solved by the technique of dynamic programming similar to [13]. However, such a solution is of high computational complexity due to "the curse of dimensionality" for dynamic programming. In addition, the resulting solution will not provide any insight to the structure of the optimal power allocation for an energy harvesting transmitter. Therefore, in this paper, we take an alternative approach by first solving the off-line optimization for (5), assuming that all the energy arrival time $t_{i}$ 's and amount $E_{i}$ 's are a priori known at the transmitter in each block transmission, and then based on the structure of the off-line optimal solution, devising online algorithms for the practical setup with only causal ESI known at the transmitter.

For the off-line optimization of (5), it is easy to see that the objective function is concave; however, the constraint is nonconvex in general since $P_{\text {total }}(t)$ in (3) is a concave function of $P(t)$ if $\alpha>0$. As a result, the problem is in general non-convex and thus cannot be solved by standard convex optimization techniques. In the next section, we will propose an efficient solution for this problem by exploiting its special structure.

Remark 2.1: It is worth noting that for the off-line optimization, (5) can be shown to be convex if $\alpha=0$. In this case, similar problems to (5) have been studied in the literature [12], [13], in which the throughput-optimal power allocation $P(t)$ was shown to follow a non-decreasing piecewise-constant (staircase) function over $t$. This power allocation can be interpreted as maximizing the SE of the point-to-point AWGN channel subject to the new energy harvesting power constraint. As will be shown later in this paper, the non-ideal circuit power with $\alpha>0$ will change the optimal power allocation for this problem considerably.

\section{OFF-Line OptimizATION}

In this section, we solve the off-line optimization problem in (5) with the non-ideal circuit power, i.e., $\alpha>0$.

\section{A. Reformulated Problem}

First, we give the following lemmal2

Lemma 3.1: During any $i$ th epoch $\left(t_{i-1}, t_{i}\right], i=1, \ldots, N$, the optimal solution for (5) is given by $P(t)=P_{i}>0$ for the portion of time $\mathcal{T}_{i}^{\text {on }} \subseteq\left(t_{i-1}, t_{i}\right]$, and $P(t)=0$ for the remaining time $\mathcal{T}_{i}^{\text {off }} \subseteq\left(t_{i-1}, t_{i}\right]$, where $\mathcal{T}_{i}^{\text {on }} \cap \mathcal{T}_{i}^{\text {off }}=\phi$ and $\mathcal{T}_{i}^{\text {on }} \cup \mathcal{T}_{i}^{\text {off }}=\left(t_{i-1}, t_{i}\right]$.

Proof: See Appendix A

According to Lemma 3.1 and by denoting the constant transmit power $P_{i}>0$ for the "on" period with length $0 \leq l_{i}^{\text {on }} \leq L_{i}$ in the $i$ th epoch, (5) can be reformulated as

$$
\begin{aligned}
\max _{\left\{P_{i}\right\},\left\{l_{i}^{\text {on }}\right\}} & \sum_{i=1}^{N} l_{i}^{\text {on }} R\left(P_{i}\right) \\
\text { s.t. } & P_{i}>0, i=1, \ldots, N \\
& 0 \leq l_{i}^{\text {on }} \leq L_{i}, i=1, \ldots, N \\
& \sum_{j=1}^{i}\left(P_{j}+\alpha\right) l_{j}^{\text {on }} \leq \sum_{j=0}^{i-1} E_{j}, i=1, \ldots, N .
\end{aligned}
$$

However, the above problem is still non-convex due to the coupling between $P_{i}$ 's and $l_{i}^{\text {on }}$ 's. In the following, we first solve this problem for the special case of $N=1$ and then generalize the solution to the case with $N \geq 1$.

\footnotetext{
${ }^{2}$ We thank the anonymous reviewer who brought our attention to [15], in which an alterative proof for Lemma 3.1 is given based on a calculus approach.
} 


\section{B. Single-Epoch Case with $N=1$}

In the single-epoch case with $N=1$, the problem in 6 is reduced to

$$
\begin{aligned}
\max _{l_{1}^{\text {on }}, P_{1}} & l_{1}^{\text {on }} R\left(P_{1}\right) \\
\text { s.t. } & P_{1}>0 \\
& 0 \leq l_{1}^{\text {on }} \leq T \\
& l_{1}^{\text {on }}\left(P_{1}+\alpha\right) \leq E_{0} .
\end{aligned}
$$

The solution of the above problem is given in the following proposition.

Proposition 3.1: The optimal solution $P_{1}^{*}$ and $l_{1}^{\text {on* }}$ for $(7)$ is expressed as

$$
\begin{gathered}
P_{1}^{*}=\max \left(P_{e e}, \frac{E_{0}}{T}-\alpha\right) \\
l_{1}^{\text {on } *}=\frac{E_{0}}{P_{1}^{*}+\alpha}
\end{gathered}
$$

where $P_{e e}$ is given by

$$
P_{e e}=\underset{P_{1}>0}{\arg \max } \frac{R\left(P_{1}\right)}{P_{1}+\alpha} .
$$

Proof: See Appendix B

It is worth noting that $P_{e e}$ given in 10 is the optimal power allocation that maximizes the EE of the AWGN channel under the non-ideal circuit power model as shown in [7]. From Proposition 3.1 it follows that if $P_{e e}>\frac{E_{0}}{T}-\alpha$, we have $P_{1}^{*}=P_{e e}, l_{1}^{\text {on } *}<T$ and $l_{1}^{\text {off } *}=T-l_{1}^{\text {on } *}>0$, which corresponds to an on-off transmission. However, if $P_{e e} \leq \frac{E_{0}}{T}-\alpha$, we have $P_{1}^{*}=\frac{E_{0}}{T}-\alpha, l_{1}^{\text {on* }}=T$ and $l_{1}^{\text {off } *}=0$, which corresponds to a continuous transmission. We will see in the next subsection that the EE-maximizing power allocation $P_{e e}$ plays an important role in the general case with $N \geq 1$. Also note that the right-hand side (RHS) of (10) is a quasiconcave function of $P_{1}$ since it is concave-over-linear [17]; thus, $P_{e e}$ can be efficiently obtained by a simple bisection search [17].

\section{Multi-Epoch Case with $N \geq 1$}

Inspired by the solution for the single-epoch case, we derive the optimal solution for (6) in the general case with $N \geq 1$, as given by the following theorem.

Theorem 3.1: The optimal solution of (6), denoted by $\left[P_{1}^{*}, \ldots, P_{N}^{*}\right]$ and $\left[l_{1}^{\text {on* }}, \ldots, l_{N}^{\text {on* } *}\right]$, is obtained as follows. Denoting

$$
\begin{aligned}
& i_{e e, 0}=0, \\
& i_{e e, j}=\min \left\{i \mid \frac{\sum_{k=i_{e e, j-1}}^{i-1} E_{k}}{\sum_{k=i_{e e, j-1}+1}^{i} L_{k}}-\alpha \leq P_{e e},\right. \\
&\left.\quad i=i_{e e, j-1}+1, \ldots, N\right\}, j \geq 1, \\
& J=\arg \max _{j \geq 0} i_{e e, j}, \\
& i_{e e}=i_{e e, J},
\end{aligned}
$$

the optimal transmit power for epochs $1, \ldots, i_{e e}$ is given by

$$
P_{i}^{*}=P_{e e}, i=1, \ldots, i_{e e}
$$

and the optimal on-period $l_{i}^{\text {on* }}, i=1, \ldots, i_{e e}$, is any set of non-negative values satisfying

$$
\begin{gathered}
\left(P_{e e}+\alpha\right) \sum_{i=1}^{i_{e e}} l_{i}^{\text {on } *}=\sum_{i=0}^{i_{e e}-1} E_{i} \\
\left(P_{e e}+\alpha\right) \sum_{i=1}^{j} l_{i}^{\text {on } *} \leq \sum_{i=0}^{j-1} E_{i}, j=1, \ldots, i_{e e} .
\end{gathered}
$$

Moreover, for epochs $i_{e e}+1, \ldots, N$, the optimal solution is given by

$$
\begin{gathered}
l_{i}^{\text {on } *}=L_{i}, i=i_{e e}+1, \ldots, N \\
P_{i}^{*}=\frac{\sum_{k=n_{i-1}}^{n_{i}-1} E_{k}}{\sum_{k=n_{i-1}+1}^{n_{i}} L_{k}}-\alpha, i=i_{e e}+1, \ldots, N
\end{gathered}
$$

where

$$
\begin{aligned}
& n_{i_{e e}}=i_{e e}, \\
& n_{i}=\arg \min _{j: n_{i-1}+1 \leq j \leq N}\left\{\frac{\sum_{k=n_{i-1}}^{j-1} E_{k}}{\sum_{k=n_{i-1}+1}^{j} L_{k}}-\alpha\right\}, \\
& i=i_{e e}+1, \ldots, N .
\end{aligned}
$$

Proof: See Appendix C

It is interesting to take note that the optimal transmission policy given in Theorem 3.1 has a two-phase structure, which is explained as follows in more details.

- $0<t \leq t_{i_{e}}$ : In the first phase, the optimal transmission is an on-off one with a constant power $P_{e e}$ for all the on-periods. Note that $P_{e e}$ is the EE-maximizing power allocation given in 10 . Also note that the optimal on-periods $l_{i}^{\text {on* }}, i=1, \ldots, i_{e e}$, may not be unique provided that they satisfy the conditions given in (13) and (14). Without loss of generality, we assume that in each epoch, the transmitter chooses to be on initially with power $P_{e e}$ provided that its stored energy is not used up, i.e., $l_{1}^{\text {on* }}=\min \left(E_{0} /\left(P_{e e}+\alpha\right), L_{1}\right), l_{2}^{\text {on* }}=$ $\min \left(\left(E_{1}+E_{0}-\left(P_{e e}+\alpha\right) l_{1}^{\text {on* }}\right) /\left(P_{e e}+\alpha\right), L_{2}\right)$, and so on.

- $t_{i_{e e}}<t \leq T$ : In the second phase, a continuous transmission is optimal. Since $l_{i}^{\text {on } *}=L_{i}, i=i_{e e}+1, \ldots, N$, the problem in (6) for $i=i_{e e}+1, \ldots, N$, is reduced to

$$
\begin{aligned}
& \max _{\left\{P_{i}\right\}} \sum_{i=i_{e e}+1}^{N} L_{i} R\left(P_{i}\right) \\
& \text { s.t. } P_{i}>0, i=i_{e e}+1, \ldots, N \\
& \sum_{j=i_{e e}+1}^{i} L_{j} P_{j} \leq \sum_{j=i_{e e}}^{i-1} E_{j}-\sum_{j=i_{e e}+1}^{i} L_{j} \alpha, \\
& i=i_{e e}+1, \ldots, N .
\end{aligned}
$$

The optimal solution for the above problem has been shown in [12], [13] to follow a non-decreasing piecewiseconstant (staircase) function, which is given in (16). It is worth noting that the staircase power allocation achieves the maximum SE for an equivalent AWGN channel subject to a sequence of energy harvesting power constraints 
(modified to take into account the circuit power $\alpha$ ) for $i=i_{e e}+1, \ldots, N$.

From the above discussion, it is revealed that for the throughput maximization in an energy harvesting transmission system subject to the non-ideal circuit power, the optimal transmission unifies both the EE and SE maximization policies independently developed in [7] and [12], [13], respectively. To summarize, one algorithm for solving the problem in 6) for the general case of $N \geq 1$ is given in Table $\mathrm{I}$.

TABLE I

Optimal OfF-Line Policy For Single-Channel CASE

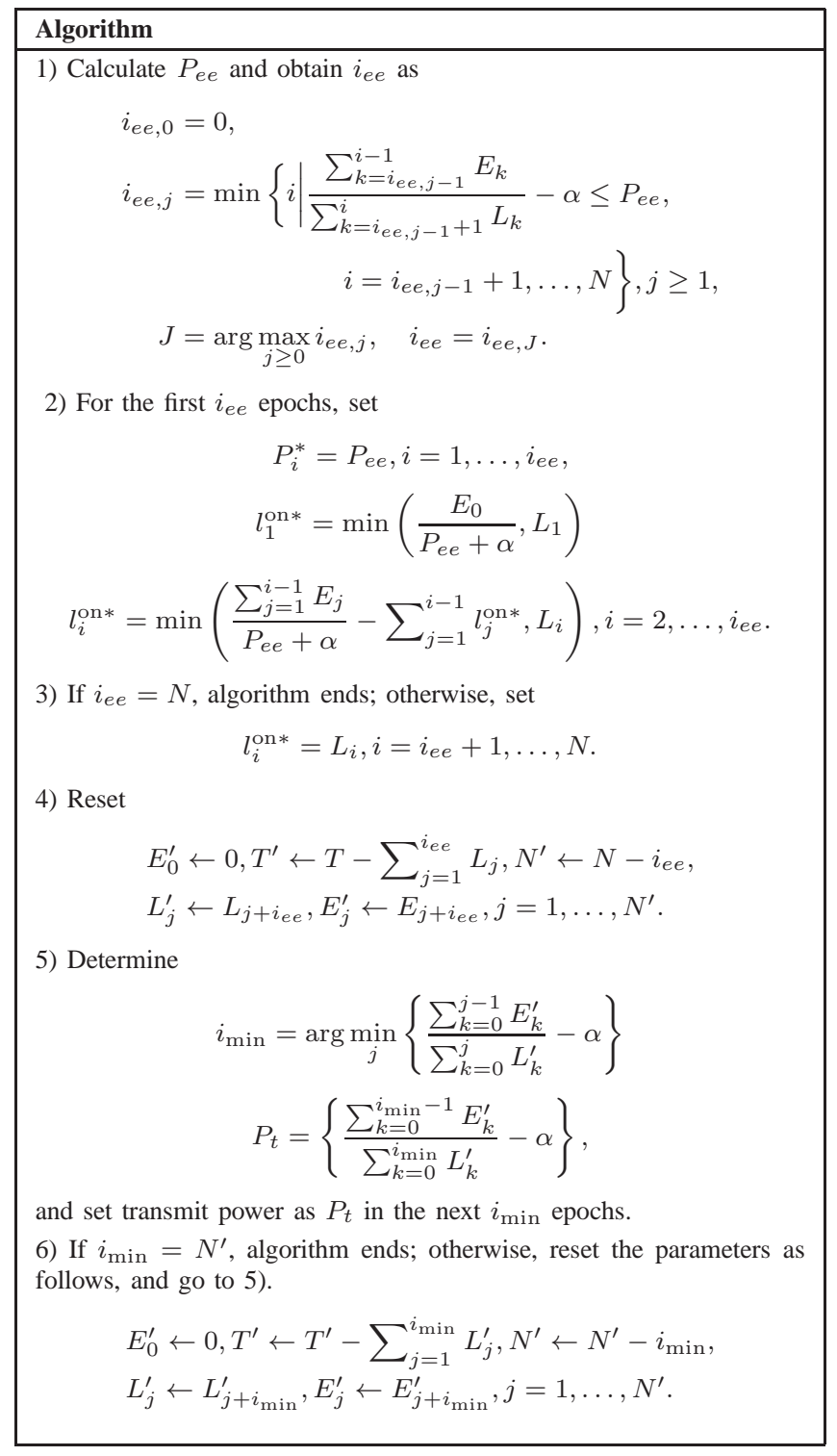

Remark 3.1: We discuss some implementation issues on energy harvesting communication systems with the optimal transmit power allocation given in Theorem 3.1. It is worth noting that in practical wireless systems, the duration of a communication block is usually on the order of millisecond, while the energy harvesting process evolves at a much slower speed, e.g., solar and wind power typically remains constant over windows of seconds. As a result, each epoch between any two consecutive energy arrivals in our model (during which the optimal power policy in Theorem 3.1 allocates a constant power) can be assumed to be sufficiently long, thus containing many communication blocks. In each communication block, pilot signals can be transmitted to help estimate the signal power at the receiver, which may change from one epoch to another due to transmit power adaptation; thus, the transmission rate in (2) is practically achievable with AMC at the transmitter and coherent detection at the receiver.

Example 3.1: To illustrate the optimal two-phase transmission given in Theorem 3.1, we consider an example of a band-limited AWGN channel with bandwidth $W=1 \mathrm{MHz}$ and the noise power spectral density $N_{0}=10^{-16}$ Watts-per$\mathrm{Hz}(\mathrm{W} / \mathrm{Hz})$. We assume that the attenuation power loss from the transmitter to the receiver is $h=-80 \mathrm{~dB}$. Considering the channel capacity with $\Gamma=1$, we thus have $R(P)=$ $W \log _{2}\left(1+\frac{P h}{\Gamma N_{0} W}\right)=\log _{2}(1+100 P)$ Mbps. It is assumed that the energy arrives at time instants $[0,4,6,11,14,16,18] \mathrm{sec}$, and the corresponding energy values are $[0.5,0.5,0.5,1,0.5$, $0.75,0.5] J o u l e$, as shown in Fig. 2 It is also assumed that $T=20$ secs and the circuit power is $\alpha=115.9 \mathrm{~mW}$. Under this setup, we compute $P_{e e}=79.2 \mathrm{~mW}$. In Fig. 2, we compare the optimal allocation of the total consumed transmitter power by the algorithm in Table I with that obtained by the algorithm given in [12], [13]. Note that the algorithm in [12] or [13] solves (6) in the special case with the ideal circuit power $\alpha=0$. Here, we apply this algorithm to obtain a suboptimal power allocation with $\alpha>0$, by assuming that the transmitter is always on, i.e., $l_{i}^{\text {on }}=L_{i}, i=1, \ldots, N$. As observed in Fig. 2 a), the optimal power allocation has a two-phase structure, i.e., an on-off transmission with transmit power $P_{e e}$ when $0<t \leq t_{3}$ followed by a continuous transmission with nondecreasing staircase power allocation when $t_{3}<t \leq T$, which is in accordance with Theorem 3.1. In contrast, as observed in Fig. 2(b), the suboptimal power allocation by the algorithm in [12] or [13] with the transmitter always on results in a continuous transmission with non-decreasing staircase power allocation during the entire block i.e. $0<t \leq T$. In addition, it can be shown that the proposed optimal solution achieves the total throughput $63.14 \mathrm{Mbits}$, while the suboptimal solution achieves only 55.80Mbits, over $T=20$ secs.

\section{Multi-Channel Optimization}

In this section, we extend the optimal off-line power allocation for the single-channel case to the general case with multiple parallel AWGN channels subject to a total energy harvesting power constraint and the non-ideal circuit power consumption at the transmitter. The multi-channel setup is applicable when the communication channel is decomposable into orthogonal channels by joint transmitter and receiver signal processing such as OFDM (orthogonal frequency division multiplexing) and/or MIMO (multiple-input multiple-output).

Without loss of generality, we assume a power vector $\mathbf{Q}(t)=\left[Q_{1}(t), \ldots, Q_{K}(t)\right] \succeq 0, t \in(0, T]$, with each element denoting the power allocation over time in one of a total $K$ parallel AWGN channels, where $\mathbf{Q}(t) \succeq 0$ denotes that $\mathbf{Q}(t)$ is elementwise no smaller than zero. We also assume a sumthroughput over the $K$ channels, denoted by $C(t)=R(\mathbf{Q}(t))$, which satisfies 

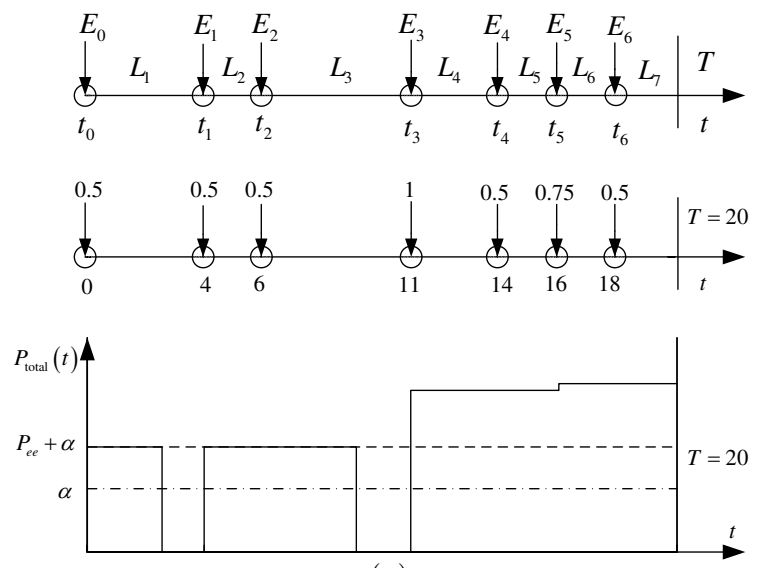

(a)

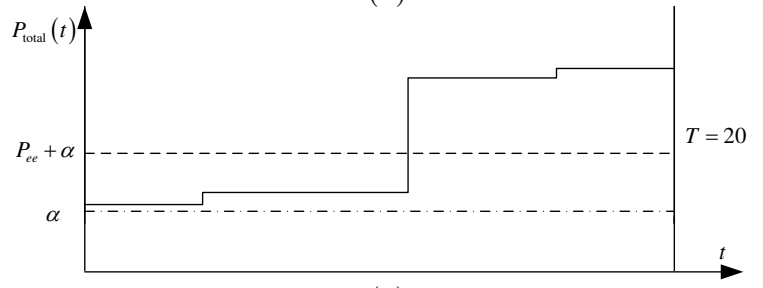

(b)

Fig. 2. Power allocation by off-line policies: (a) the optimal off-line policy; and (b) the off-line policy in [12], [13].

1) $R(\mathbf{Q}(t)) \geq 0, \forall \mathbf{Q}(t) \succeq 0$, and $R(\mathbf{0})=0$;

2) $R(\mathbf{Q}(t))$ is a strictly joint concave function over $\mathbf{Q}(t) \succeq$ 0 ;

3) $R(\mathbf{Q}(t))$ is a monotonically increasing function with respect to each argument in $\mathbf{Q}(t)$ i.e. $Q_{k}(t) \geq 0, k=$ $1, \ldots, K$.

An example of the above multi-channel sum-throughput is the sum-rate over $K$ parallel AWGN channels achieved by joint $\mathrm{AMC}$, which is given by

$$
R(\mathbf{Q}(t))=W \sum_{k=1}^{K} \log _{2}\left(1+\frac{h_{k} Q_{k}(t)}{\Gamma W N_{0}}\right)
$$

where $h_{k} \geq 0$ denotes the channel power gain of the $k$ th channel. Similar to (3) in the single-channel case, by taking into account the non-ideal circuit power $\alpha$, the total power consumed at the transmitter for the multi-channel case is modeled by

$$
Q_{\text {total }}(t)= \begin{cases}\sum_{k=1}^{K} Q_{k}(t)+\alpha, & \sum_{k=1}^{K} Q_{k}(t)>0 \\ 0 & \sum_{k=1}^{K} Q_{k}(t)=0 .\end{cases}
$$

Then the throughput maximization problem over a finite horizon $T$ in the multi-channel case is formulated as

$$
\begin{aligned}
\max _{\mathbf{Q}(t) \succeq 0} & \int_{0}^{T} R(\mathbf{Q}(t)) \mathrm{d} t \\
\text { s.t. } & \int_{0}^{t_{i}} Q_{\text {total }}(t) \mathrm{d} t \leq \sum_{j=0}^{i-1} E_{j}, i=1, \ldots, N .
\end{aligned}
$$

Similar to (5), the above problem is non-convex with $\alpha>0$ and thus cannot be solved by standard convex optimization techniques. In the following, we will apply the principle of nested optimization to convert this problem with multidimensional (vector) power optimization to an equivalent problem with only one-dimensional (scalar) power optimization, which is then optimally solvable by the algorithm in Table I for the single-channel case.

To apply the nested optimization, we first introduce an auxiliary variable $P(t)=\sum_{k=1}^{K} Q_{k}(t)$, and rewrite the objective function of 21) equivalently as

$$
\begin{aligned}
& \max _{P(t) \geq 0} \max _{\mathbf{Q}(t): Q_{k}(t) \geq 0, \forall k, \sum_{k=1}^{K} Q_{k}(t) \leq P(t)} \int_{0}^{T} R(\mathbf{Q}(t)) \mathrm{d} t \\
= & \max _{P(t) \geq 0} \int_{0}^{T} R(\mathbf{Q}(t)) \mathrm{d} t .
\end{aligned}
$$

Define the auxiliary function

$$
\bar{R}(P(t))=\underset{\mathbf{Q}(t): Q_{k}(t) \geq 0, \forall k, \sum_{k=1}^{K} Q_{k}(t) \leq P(t)}{\max } R(\mathbf{Q}(t))
$$

for which it can be easily verified that the maximum is attained when $\sum_{k=1}^{K} Q_{k}(t)=P(t)$. Thus, without loss of generality, we can rewrite 21 equivalently as

$$
\begin{aligned}
\max _{P(t) \geq 0} & \int_{0}^{T} \bar{R}(P(t)) \mathrm{d} t \\
\text { s.t. } & \int_{0}^{t_{i}} P_{\text {total }}(t) \mathrm{d} t \leq \sum_{j=0}^{i-1} E_{j}, \quad i=1, \ldots, N
\end{aligned}
$$

where

$$
P_{\text {total }}(t)=\left\{\begin{array}{cc}
P(t)+\alpha, & P(t)>0 \\
0, & P(t)=0 .
\end{array}\right.
$$

Thus, the original problem with vector power optimization is converted by the nested optimization to an equivalent problem with only scalar power optimization. Thereby, we can first solve (24) to get the optimal solution of $P(t)$, and then with the obtained $P(t)$ solve (23) to find the optimal solution of $\mathbf{Q}(t)$ for (21). Since the problem in 23) is a convex optimization problem, it can be solved by standard techniques e.g. the Lagrange duality method [17] (in the special case of the sumrate given in 19], the optimal solution can be obtained by the well-known "water-filling" algorithm [17]).

In order to solve (24), we first give the following proposition.

Proposition 4.1: The function $\bar{R}(P(t))$ satisfies the following properties:

1) $\bar{R}(P(t)) \geq 0, \forall P(t) \geq 0$, and $\bar{R}(0)=0$;

2) $\bar{R}(P(t))$ is a strictly concave function over $P(t) \geq 0$;

3) $\bar{R}(P(t))$ is a monotonically increasing function over $P(t) \geq 0$.

Proof: See Appendix D

Since $\bar{R}(P(t))$ satisfies the same conditions as $R(P(t))$ for the single-channel case, it follows that (24) can be similarly 
solved by the algorithm in Table I, with one minor modification: the EE-maximizing power allocation in the multi-channel case needs to be obtained as

$$
\begin{aligned}
P_{e e} & =\arg \max _{P>0} \frac{\bar{R}(P)}{P+\alpha} \\
& =\arg \max _{P>0} \frac{\operatorname{QQ}: Q_{k} \geq 0, \forall k, \sum_{k=1}^{K} Q_{k} \leq P}{P+\alpha} R(\mathbf{Q})
\end{aligned}
$$

Since the maximum in the above problem is attained by $\sum_{k=1}^{K} Q_{k}=P$, the optimal solution of $\mathbf{Q}$ is obtained as

$$
\mathbf{Q}^{e e}=\arg \max _{\mathbf{Q}: Q_{k} \geq 0, \forall k, \sum_{k=1}^{K} Q_{k} \leq P} \frac{R(\mathbf{Q})}{\sum_{k=1}^{K} Q_{k}+\alpha}
$$

with $\mathbf{Q}^{e e}=\left[Q_{1}^{e e}, \ldots, Q_{K}^{e e}\right]$, and

$$
P_{e e}=\sum_{k=1}^{K} Q_{k}^{e e}
$$

Since the RHS of (27) is a quasi-concave function, this problem is quasi-convex and thus can be efficiently solved by the bisection method [17]. Here we omit the detail for brevity.

To summarize, the algorithm for solving (21) for the multichannel case is given in Table II.

TABLE II

Optimal OfF-Line Policy For Multi-Channel Case

Algorithm
1) Obtain $P_{e e}$ by solving [27) and [28; apply the algorithm in Table
[to obtain the solution $P^{*}(t)$ for 24].
2) With the obtained $P^{*}(t)$, solve [23] to obtain the solution $\mathbf{Q}^{*}(t)$
for 21].

\section{ONLINE Algorithm}

In the previous two sections, we have studied the optimal off-line policies assuming the non-causal ESI at the transmitter, which provide the throughput upper bound for all online policies. In this section, we will address the practical online case with only the causal (past and present) ESI assumed to be known at the transmitter. In particular, we will propose an online policy based on the structure of the optimal offline policy obtained previously in Section [II. Due to the space limitation, we will only consider the single-channel case for the study of online algorithms, while similar results can be obtained for the general multi-channel case, based on the optimal off-line policy given in Section IV

\section{A. Proposed Online Algorithm}

For the purpose of exposition, we assume that the harvested energy is modeled by a compound Poisson process, where the number of energy arrivals over a horizon $T$ follows a Poisson distribution with mean $\lambda_{e} T$ and the energy amount in each arrival is independent and identically (i.i.d.) distributed with mean $\bar{E}$. It is assumed that $\lambda_{e}$ and $\bar{E}$ are known at the transmitter.
We propose an online power allocation algorithm based on the structure of the optimal off-line solution revealed in Theorem 3.1 Specifically, considering the start time of each block, from Theorem 3.1 we obtain the closed-form solution for the optimal off-line power allocation at $t=0$ in the following proposition.

Proposition 5.1: Suppose there are $N-1$ energy arrivals in $\overline{(0, T) \text { with }} N \geq 1$, the optimal off-line power allocation solution for (6) at $t=0$ is given by

$$
P^{*}(0)=\max \left(\min _{i=1, \ldots, N}\left(\frac{\sum_{k=0}^{i-1} E_{k}}{\sum_{k=1}^{i} L_{k}}-\alpha\right), P_{e e}\right) .
$$

Proof: See Appendix E

Note that in 29), $E_{0}$ is available at the transmitter at $t=0$, while $N, E_{i}, i=1, \ldots, N-1$, and $L_{i}, i=1, \ldots, N$, are all unknown at the transmitter due to the causal ESI. As a result, we cannot compute $P^{*}(0)$ in 29) at $t=0$ for the online policy. Nevertheless, we can approximate the expression of $P^{*}(0)$ based on the statistical knowledge of the energy arrival process, i.e., $\lambda_{e}$ and $\bar{E}$, as follows.

Denote

$$
\frac{\sum_{k=0}^{i-1} E_{k}}{\sum_{k=1}^{i} L_{k}}=\frac{E_{0}+\sum_{k=1}^{i-1} E_{k}}{\sum_{k=1}^{i} L_{k}}=\frac{E_{0}}{\sum_{k=1}^{i} L_{k}}+\frac{\sum_{k=1}^{i-1} E_{k}}{\sum_{k=1}^{i} L_{k}} .
$$

For any $i \leq N, \sum_{k=1}^{i-1} E_{k}$ is the total energy harvested during $\left(0, t_{i}\right)$ and $\sum_{k=1}^{i} L_{k}=t_{i}$. We thus have

$$
\frac{\sum_{k=1}^{i-1} E_{k}}{\sum_{k=1}^{i} L_{k}} \approx \frac{\lambda_{e} t_{i} \bar{E}}{t_{i}}=\lambda_{e} \bar{E}, \forall 1<i \leq N,
$$

where the approximation becomes exact when $t_{i} \rightarrow \infty$.

Using (30), we can approximate $\frac{\sum_{k=0}^{i-1} E_{k}}{\sum_{k=1}^{i} L_{k}}$ as $\frac{E_{0}}{\sum_{k=1}^{i} L_{k}}+$ $\lambda_{e} \bar{E}$, and

$$
\begin{aligned}
& \min _{i=1, \ldots, N}\left(\frac{\sum_{k=0}^{i-1} E_{k}}{\sum_{k=1}^{i} L_{k}}-\alpha\right) \\
\approx & \min _{i=1, \ldots, N}\left(\frac{E_{0}}{\sum_{k=1}^{i} L_{k}}+\lambda_{e} \bar{E}-\alpha\right)=\frac{E_{0}}{T}+\lambda_{e} \bar{E}-\alpha,
\end{aligned}
$$

and then obtain

$$
P^{*}(0) \approx \max \left(\frac{E_{0}}{T}+\lambda_{e} \bar{E}-\alpha, P_{e e}\right) .
$$

Since $E_{0}, \lambda_{e}$ and $\bar{E}$ are all known at the transmitter at $t=0$, (31) can be computed in real time.

For any $0<t<T$, by denoting the stored energy as $E_{s}(t)$ with $E_{s}(0)=E_{0}$, we can view the online throughput maximization at time $t$ over the remaining time $T-t$ to have an initial stored energy $E_{0}=E_{s}(t)$. Therefore, by replacing $T$ and $E_{0}$ in (31) as $T-t$ and $E_{s}(t)$, respectively, we obtain the following online transmit power allocation policy:

$$
P_{\text {online }}(t)= \begin{cases}\max \left(\frac{E_{s}(t)}{T-t}+\lambda_{e} \bar{E}-\alpha, P_{e e}\right), & E_{s}(t)>0 \\ 0, & E_{s}(t)=0\end{cases}
$$

for any $t \in[0, T)$. 


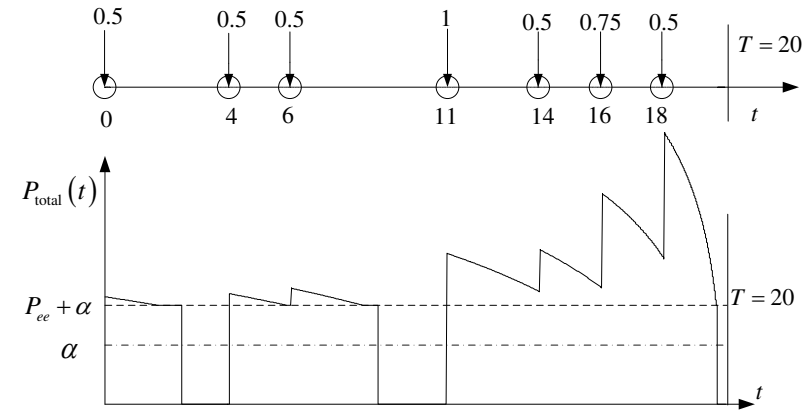

Fig. 3. Power allocation by the proposed online policy.

The online policy in 32 provides some useful insights. Note that $\frac{E_{s}(t)}{T-t}+\lambda_{e} \bar{E}-\alpha$ can be viewed as the "expected" available transmit power for the remaining time in each block, which can be negative for some $t$ if $\frac{E_{s}(t)}{T-t}+\lambda_{e} \bar{E}<\alpha$. Thus, if this value is less than the EE-maximizing power allocation $P_{e e}$, the transmitter should transmit with $P_{e e}$ to save energy; however, if the inequality is reversed, the transmitter should transmit more power to maximize the SE. Moreover, as compared to the optimal off-line power allocation for the single-epoch case given in Proposition 3.1, we see that the online policy (32) bears a similar structure, by noting that $E_{0} / T-\alpha$ in (8) for the single-epoch case is also the available transmit power for the remaining time in each block. Last, it is worth remarking that the online power allocation policy in (32) is expressed as a function of the continuous time for convenience; however, in practice, this policy needs to be implemented in discrete time steps by properly quantizing the continuous-time function. The time step needs to be carefully chosen in implementation: On one hand, it is desirable to use smaller step values to achieve higher quantization accuracy for energy saving, while on the other hand, the time step needs to be sufficiently large, i.e., at least larger than one communication block (cf. Remark 3.11) so that the receiver can have a timely estimate of any transmit power adjustment.

Example 5.1: To illustrate the proposed online power allocation policy in (32), we consider the same channel setup and harvested energy process for the off-line case in Example 3.1 (cf. Fig. 2). In Fig. 3, we show the total transmitter power consumption by the proposed online policy assuming that the exact average harvested power $\lambda_{e} \bar{E}=\left(\sum_{i=1}^{N-1} E_{i}\right) / T=$ $187.5 \mathrm{~mW}$ is known at the transmitter. It is observed that the online power allocation is no more piecewise-constant like the optimal off-line power allocation in Fig. 2 (a). Nevertheless, it is also observed that these two policies result in some similar power allocation patterns, i.e., starting with an onoff power allocation followed by a non-decreasing (in the sense of average power profile for the online policy case) power allocation. This suggests that the proposed online policy captures the essential features of the optimal off-line policy. Moreover, it can be shown that the proposed online policy achieves the total throughput $61.61 \mathrm{Mbits}$ over $T=20 \mathrm{secs}$, which is only $1.53 \mathrm{Mbits}$ from $63.14 \mathrm{Mbits}$ of the optimal off-line policy. In addition, it can be verified that the total

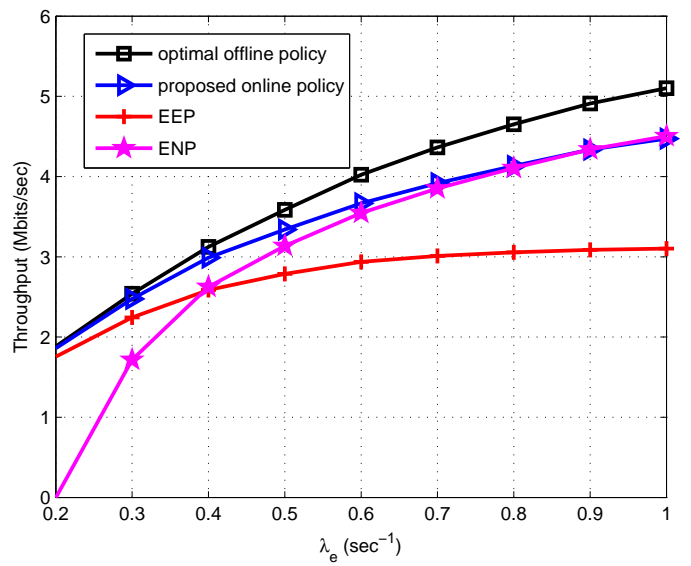

Fig. 4. Average throughput versus the energy arrival rate $\lambda_{e}$ with $\bar{E}=0.5 \mathrm{~J}$ and $T=20$ secs.

throughput obtained by the proposed online policy is very robust to the assumed average harvested power value $\lambda_{e} \bar{E}$. For example, by setting $\lambda_{e} \bar{E}$ to be $150 \mathrm{~mW}$ or $200 \mathrm{~mW}$, the proposed online policy obtains the throughput $61.38 \mathrm{Mbits}$ and $61.60 \mathrm{Mbits}$, respectively, which is a very small loss in either case.

\section{Simulation Results}

In the section, we compare the performance of the proposed online policy with the performance upper bound achieved by the optimal off-line policy under a stochastic energy harvesting setup modeled by the compound Poisson process. The amount of energy in each energy arrival is assumed to be independent and uniformly distributed between 0 and $2 \bar{E}$. For the purpose of comparison, we also consider two alternative heuristically designed online power allocation policies given as follows.

- Energy Efficient Policy (EEP): In this online policy, the transmitter transmits with the EE-maximizing power allocation $P_{e e}$ given in (10) provided that there is a nonzero stored energy, i.e.,

$$
P_{\mathrm{EEP}}(t)= \begin{cases}P_{e e}, & E_{s}(t)>0 \\ 0, & E_{s}(t)=0\end{cases}
$$

for any $t \in[0, T)$.

- Energy Neutralization Policy (ENP): This online policy transmits with a constant power that satisfies the longterm energy consumption constraint if there is available stored energy, i.e.,

$$
P_{\mathrm{ENP}}(t)= \begin{cases}\lambda_{e} \bar{E}-\alpha, & E_{s}(t)>0 \\ 0, & E_{s}(t)=0\end{cases}
$$

for any $t \in[0, T)$. Note that in the above we have assumed that $\lambda_{e} \bar{E}>\alpha$.

First, we consider a single AWGN channel in Figs. 4 and 5 with the same channel parameters as for Example 3.1 In Fig. 4 we show the average throughput over $T=20$ secs versus $\lambda_{e}$, with $\bar{E}=0.5 \mathrm{Joule}(\mathrm{J})$. It is observed that when $\lambda_{e}$ is small, the proposed online policy and EEP obtain similar performance as the optimal off-line policy. Since the average 


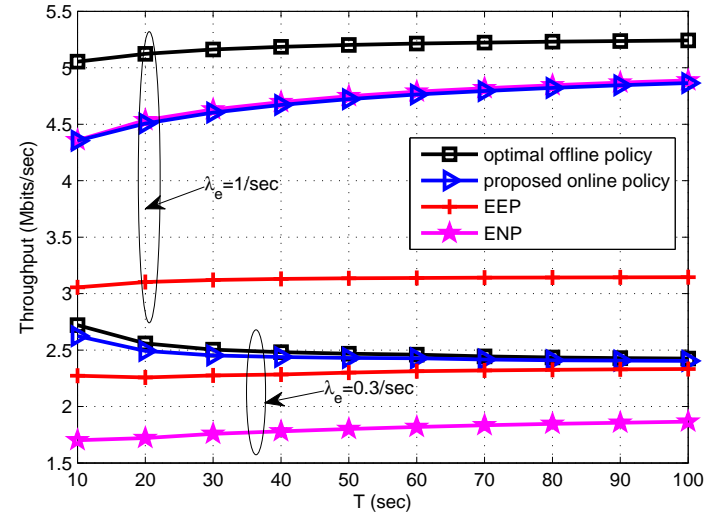

Fig. 5. Average throughput versus the block duration $T$ with $\bar{E}=0.5 \mathrm{~J}$.

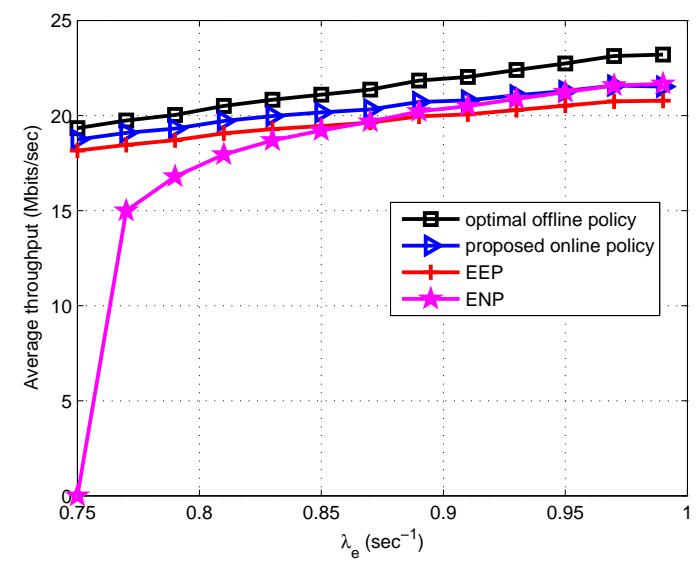

Fig. 6. Average throughput in a single-cell OFDMA downlink system with renewable powered BS versus the energy arrival rate $\lambda_{e}$ with $\bar{E}=200 \mathrm{~J}$ and $T=20$ secs.

harvested energy is small when $\lambda_{e}$ is small, it is more likely that $P_{e e}$ is greater than both $\frac{E_{s}(t)}{T-t}+\lambda_{e} E-\alpha$ (c.f. 32) and $\frac{\sum_{k=0}^{i-1} E_{k}}{\sum_{k=1}^{i} L_{k}}-\alpha, \forall i=1, \ldots, N$ (c.f. 29). Thus, both the proposed online and off-line policies choose to transmit with $P_{e e}$ during the "on" periods to save energy. However, for ENP, the transmit power level deviates from $P_{e e}$ and thus significant amount of energy is consumed due to the nonideal circuit power; as a result, the achievable throughput is almost zero. As $\lambda_{e}$ increases, the throughput gap between the optimal off-line policy and all online policies enlarges, and the performance of EEP degenerates severely. Moreover, ENP is observed to obtain a similar performance as the proposed online policy. This is because in this case, $\frac{E_{s}(t)}{T-t}$ is negligibly small as compared with $\lambda_{e} E$, and as a result the proposed online policy degenerates to ENP.

Fig. 5 shows the average throughput versus $T$, for two different values of $\lambda_{e}=0.3 / \mathrm{sec}$ and $1 / \mathrm{sec}$, with $\bar{E}=0.5 \mathrm{~J}$. In both cases of $\lambda_{e}$, the proposed online policy is observed to perform close to the optimal off-line policy, for all values of $T$. With small value of $\lambda_{e}$, i.e., $\lambda_{e}=0.3 / \mathrm{sec}$, EEP performs much better than ENP since it is more energy efficient, while with a larger value of $\lambda_{e}$, i.e., $\lambda_{e}=1 /$ sec, the reverse becomes true, which can be similarly explained as for Fig. 4

Furthermore, for the multi-channel scenario, in Fig. 6 we evaluate the average throughput of a single-cell downlink system with the base station (BS) powered by energy harvesting. It is assumed that the BS covers a circular area with radius 1000 meters, and serves $K$ users whose locations are generated following a spatial homogeneous Poisson point process (HPPP) with density $10^{-6}$ users $/ \mathrm{m}^{2}$. Consider a simplified channel model without fading, in which the channel power gain of each user from the BS is determined by a pathloss model $c_{0}\left(\frac{r}{r_{0}}\right)^{-\zeta}$, where $c_{0}=-60 \mathrm{~dB}$ is a constant equal to the pathloss at a reference distance $d_{0}=10 \mathrm{~m}$, and $\zeta=3$ is the pathloss exponent. Assuming an OFDMA (orthogonal frequency division multiplexing access) based user multiple access, a total bandwidth $W=5 \mathrm{MHz}$ is equally allocated to the $K$ users. The noise power spectral density at each user receiver is set as $N_{0}=-174 \mathrm{dBm} / \mathrm{Hz}$ and $\Gamma=1$ is assumed. The circuit power at the BS is set as $\alpha=60$ Watt. Fig. 6 shows the average throughput versus $\lambda_{e}$ with $\bar{E}=200 \mathrm{~J}$ and an energy scheduling period $T=20$ secs. It is observed that the proposed online policy always performs better than EEP. With small values of $\lambda_{e}$, i.e., $\lambda_{e} \leq 0.8 / \mathrm{sec}$, ENP preforms clearly worse than the proposed online algorithm, while when $\lambda_{e} \geq 0.95 / \mathrm{sec}$, both schemes perform similarly. This observation is expected as can be similarly explained for Fig. 4.

\section{CONCluding REMARKS}

In this paper, we studied the throughput-optimal transmission policies for energy harvesting wireless transmitters with the non-ideal circuit power. We first obtained the optimal off-line solution in the single-channel case, which is shown to have a new two-phase transmission structure by unifying existing results on separately maximizing energy efficiency and spectrum efficiency. We then extended the optimal offline solution to the general case with multiple AWGN channels subject to a total energy harvesting power constraint, by the technique of nested optimization. Finally, we proposed an online algorithm based on a closed-form off-line solution. It is shown by simulations that the proposed online algorithm has a very close performance to the upper bound achieved by the optimal off-line solution, and also outperforms other heuristically designed online algorithms.

After submission of this manuscript, we become aware of one interesting related work [19] that is worth mentioning. In [19], the throughput optimization in a single-channel energy harvesting communication system with battery leakage is introduced. The impact of battery leakage is very similar to that of the non-ideal circuit power considered in this paper; as a result, the optimal transmission policy developed in [19] is similar to the one proposed in Section III of this paper. One difference is that the optimal off-line policy in [19] is developed by decoupling the general multi-epoch problem into multiple equivalent single-epoch subproblems, while the same optimal policy in this paper is derived by decoupling the problem into only two subproblems (c.f. Appendix [C). Compared with the solution in [19], the off-line policy in this paper reveals the optimal two-phase structure in which EE and 
SE optimizations are unified, and thus motivates our online policies, which are not given in [19].

\section{APPENDIX A}

PROOF OF LEMMA 3.1

Denote the length of the on-period $\mathcal{T}_{i}^{\text {on }}$ as $l_{i}^{\text {on }}$ and that of the off-period $\mathcal{T}_{i}^{\text {off }}$ as $l_{i}^{\text {off }}$, where $l_{i}^{\text {on }}+l_{i}^{\text {off }}=L_{i}$. Without loss of generality, we only need to consider the case with onperiod $\mathcal{T}_{i}^{\text {on }}=\left(t_{i-1}, t_{i-1}+l_{i}^{\text {on }}\right]$ and off-period $\mathcal{T}_{i}^{\text {off }}=\left(t_{i-1}+\right.$ $\left.l_{i}^{\text {on }}, t_{i}\right]$, since exchanging the power allocation at two different time instants in an epoch does not change the throughput and the energy constraint. Next, we prove that the transmit power should be constant during the on-period $\mathcal{T}_{i}{ }^{\text {on }}$ in an epoch by contradiction.

Suppose that the optimal allocated transmit power $\bar{P}(t)$, where $\bar{P}(t)>0, t \in\left(t_{i-1}, t_{i-1}+l_{i}^{\text {on }}\right]$ is not constant. Since $R(P(t))$ is a strictly concave function, based on Jensen's inequality, we have

$$
R\left(\frac{\int_{t_{i-1}}^{t_{i-1}+l_{i}^{\text {on }}} \bar{P}(t) \mathrm{d} t}{l_{i}^{\text {on }}}\right)>\int_{t_{i-1}}^{t_{i-1}+l_{i}^{\text {on }}} \frac{R(\bar{P}(t))}{l_{i}^{\text {on }}} \mathrm{d} t
$$

and then

$$
\begin{aligned}
& \int_{t_{i-1}}^{t_{i-1}+l_{i}^{\text {on }}} R\left(\frac{\int_{t_{i-1}}^{t_{i-1}+l_{i}^{\text {on }}} \bar{P}(t) \mathrm{d} t}{l_{i}^{\text {on }}}\right) \mathrm{d} t \\
= & l_{i}^{\text {on }} R\left(\frac{\int_{t_{i-1}}^{t_{i-1}+l_{i}^{\text {on }}} \bar{P}(t) \mathrm{d} t}{l_{i}^{\text {on }}}\right) \\
> & \int_{t_{i-1}}^{t_{i-1}+l_{i}^{\text {on }}} R(\bar{P}(t)) \mathrm{d} t .
\end{aligned}
$$

Thus, if we construct a new transmit power allocation $\hat{P}(t)$ as $\hat{P}(t)=P_{i}=\frac{\int_{t_{i-1}}^{t_{i-1}+l_{i}^{\text {on }}} \bar{P}(t) \mathrm{d} t}{l_{i}^{\text {on }}}>0, t \in\left(t_{i-1}, t_{i-1}+l_{i}^{\text {on }}\right]$, we can achieve a larger throughput than that achieved by $\bar{P}(t)$. Moreover, we verify that $\hat{P}(t)$ consumes the same total energy as $\bar{P}(t)$ in the $i$ th epoch, i.e.,

$$
\begin{aligned}
& \int_{t_{i-1}}^{t_{i-1}+l_{i}^{\text {on }}}(\hat{P}(t)+\alpha) \mathrm{d} t=l_{i}^{\text {on }}\left(P_{i}+\alpha\right) \\
= & \int_{t_{i-1}}^{t_{i-1}+l_{i}^{\text {on }}} \bar{P}(t) \mathrm{d} t+l_{i}^{\text {on }} \alpha=\int_{t_{i-1}}^{t_{i-1}+l_{i}^{\text {on }}}(\bar{P}(t)+\alpha) \mathrm{d} t .
\end{aligned}
$$

Therefore, based on 36 and 37, we conclude that $\bar{P}(t)$ cannot be optimal and thus Lemma 3.1 is proved.

\section{APPENDIX B}

\section{Proof of Proposition 3.1}

To solve (7), we note that the third inequality constraint must be met with equality by the optimal solution, since otherwise the throughput can be further improved by increasing $P_{1}$. Thus, by substituting $l_{1}^{\text {on }}=\frac{E_{0}}{P_{1}+\alpha}$ into the objective function as well as the constraint $l_{1}^{\text {on }} \leq T$, the problem becomes equivalent to finding

$$
\begin{aligned}
P_{1}^{*} & =\arg \max _{P_{1}>0, P_{1} \geq E_{0} / T-\alpha} \frac{E_{0}}{P_{1}+\alpha} R\left(P_{1}\right) \\
& =\arg \max _{P_{1}>0, P_{1} \geq E_{0} / T-\alpha} \frac{R\left(P_{1}\right)}{P_{1}+\alpha} .
\end{aligned}
$$

Consider first the following problem with the relaxed power constraint:

$$
\max _{P_{1}>0} \frac{R\left(P_{1}\right)}{P_{1}+\alpha} \text {. }
$$

This problem has been studied in [7], where the globally optimal solution is known as the EE-maximizing power allocation, denoted by $P_{e e}$. It was also shown in [7] that given $\alpha>0$, $\frac{R\left(P_{1}\right)}{P_{1}+\alpha}$ is monotonically increasing with $P_{1}$ if $0 \leq P_{1}<P_{e e}$, and monotonically decreasing with $P_{1}$ if $P_{1}>P_{e e}$. Thus, the solution of 38 is obtained as

$$
P_{1}^{*}=\max \left(P_{e e}, \frac{E_{0}}{T}-\alpha\right) .
$$

Accordingly, the optimal on-period is given by

$$
l_{1}^{\mathrm{on} *}=\frac{E_{0}}{P_{1}^{*}+\alpha} .
$$

Proposition 3.1 is thus proved.

\section{APPENDIX C ProOF OF THEOREM 3.1}

To prove Theorem 3.1 we construct the following two subproblems $\mathbb{P}_{1}$ and $\mathbb{P}_{2}$ for the power allocation optimization in the first $i_{e e}$ epochs and the last $N-i_{e e}$ epochs, respectively.

$$
\begin{aligned}
& \mathbb{P}_{1}: \max _{\left\{P_{i}\right\},\left\{l_{i}^{\text {on }}\right\}} \sum_{i=1}^{i_{e e}} l_{i}^{\text {on }} R\left(P_{i}\right), \\
& \text { s.t. } \quad 0 \leq l_{i}^{\text {on }} \leq L_{i}, i=1, \ldots, i_{e e} \\
& \\
& \sum_{j=1}^{i}\left(P_{j}+\alpha\right) l_{j}^{\text {on }} \leq \sum_{j=0}^{i-1} E_{j}, i=1, \ldots, i_{e e} .
\end{aligned}
$$

$$
\begin{aligned}
& \mathbb{P}_{2}: \max _{\left\{P_{i}\right\},\left\{l_{i}^{\text {on }}\right\}} \sum_{i=i_{e e}+1}^{N} l_{i}^{\text {on }} R\left(P_{i}\right), \\
& \text { s.t. } 0 \leq l_{i}^{\text {on }} \leq L_{i}, i=i_{e e}+1, \ldots, N \\
& \\
& \sum_{j=i_{e e}+1}^{i}\left(P_{j}+\alpha\right) l_{j}^{\text {on }} \leq \sum_{j=i_{e e}}^{i-1} E_{j}, \\
& i=i_{e e}+1, \ldots, N .
\end{aligned}
$$

We will first prove that the solution given in Theorem 3.1 is optimal for both $\mathbb{P}_{1}$ and $\mathbb{P}_{2}$, and then prove that the optimal solutions for $\mathbb{P}_{1}$ and $\mathbb{P}_{2}$ are also optimal for $[6$.

First, we prove that the solution given in (12), (13) and (14) is optimal for $\mathbb{P}_{1}$.

Consider the throughput maximization problem $\mathbb{P}_{1}$, with the arrived energy $E_{0}, \ldots, E_{i_{e e}-1}$ at time $t_{0}, \ldots, t_{i_{e e}-1}$ over the horizon $T_{1}=\sum_{k=1}^{i_{e e}} L_{k}$. We construct an auxiliary throughput maximization problem $\overline{\mathbb{P}}_{1}$ with the energy arrival 
$\sum_{k=0}^{i_{e e}-1} E_{k}, 0, \ldots, 0$ at time $t_{0}, \ldots, t_{i_{e e}-1}$ over the same horizon $T_{1}$ as follows.

$$
\begin{aligned}
\overline{\mathbb{P}}_{1}: \max _{\left\{P_{i}\right\},\left\{l_{i}^{\text {on }}\right\}} & \sum_{i=1}^{i_{e e}} l_{i}^{\text {on }} R\left(P_{i}\right), \\
\text { s.t. } & 0 \leq l_{i}^{\text {on }} \leq L_{i}, i=1, \ldots, i_{e e}, \\
& \sum_{j=1}^{i_{e e}}\left(P_{j}+\alpha\right) l_{j}^{\text {on }} \leq \sum_{j=0}^{i_{e e}-1} E_{j} .
\end{aligned}
$$

It is clear that the optimal throughput of $\overline{\mathbb{P}}_{1}$ is an upper bound on that of $\mathbb{P}_{1}$ since any feasible solution of $\mathbb{P}_{1}$ is also feasible for $\overline{\mathbb{P}}_{1}$. Note that there is no energy arrived in $t_{1}, \ldots, t_{i_{e e}-1}$ for $\overline{\mathbb{P}}_{1}$, so $\overline{\mathbb{P}}_{1}$ is indeed equivalent to a throughput maximization problem for the single-epoch case studied in Section IIIB over a horizon $T_{1}$. It can be easily verified based on (11) that $\frac{\sum_{j=0}^{i_{e e}-1} E_{j}}{T_{1}}-\alpha \leq P_{e e}$; thus, it follows after some simple manipulation that the optimal value of $\overline{\mathbb{P}}_{1}$ is $\frac{\sum_{j=0}^{i_{e e}-1} E_{j}}{P_{e e}+\alpha} \cdot R\left(P_{e e}\right)$ and is attained by $\bar{P}_{1}^{*}=\cdots=\bar{P}_{i_{e e}}^{*}=P_{e e}$ and $\sum_{j=1}^{i_{e e}} \bar{l}_{j}^{\text {on* } *}=\frac{\sum_{j=0}^{i_{e e}-1} E_{j}}{P_{e e}+\alpha}$. Meanwhile, for $\mathbb{P}_{1}$ we can always construct a feasible solution based on (12), (13) and (14) by setting

$$
\begin{aligned}
P_{j}^{*} & =P_{e e}, \forall j=1, \ldots, i_{e e} \\
l_{k}^{\text {on* }} & =L_{k}, \forall k \neq i_{e e, j}, \forall j=1, \ldots, J \\
l_{i_{e e, j}}^{\text {on } *} & =\frac{\sum_{k=i_{e e, j-1}}^{i_{e e, j}-1}}{P_{e e}+\alpha}-\sum_{k=i_{e e, j-1}+1}^{i_{e e, j}} L_{k}, \forall j=1, \ldots, J .
\end{aligned}
$$

It can be verified from (11) that the solution satisfying (45) is feasible for $\mathbb{P}_{1}$, and attains an objective value of $\frac{\sum_{j=0}^{i_{e e}-1} E_{j}}{P_{e e}+\alpha}$. $R\left(P_{e e}\right)$, which is the same as the optimal value of $\overline{\mathbb{P}}_{1}$. The gap between $\mathbb{P}_{1}$ and $\overline{\mathbb{P}}_{1}$ is thus zero, and accordingly, the solution given in (12), (13) and (14) is optimal for $\mathbb{P}_{1}$.

Second, we prove that the solution in (15), (16) and 177 is optimal for Problem $\mathbb{P}_{2}$ over the last $N-i_{e e}$ epochs. First, we show $l_{i}^{\text {on* }}=L_{i}, i=i_{e e}+1, \ldots, N$ by contradiction as follows. Suppose that the optimal solution $\hat{P}(t)$ contains an "off" period with $\left(\hat{t}^{\text {off }}, \hat{t}^{\text {off }}+\Delta \hat{t}^{\text {off }}\right) \subset\left(t_{i_{e e}}, T\right]$, i.e., $\hat{P}(t)=$ $0, t \in\left(\hat{t}^{\text {off }}, \hat{t}^{\text {off }}+\Delta \hat{t}^{\text {off }}\right)$.

Due to the definition of 111, it follows immediately that

$$
\frac{\sum_{k=i_{e e}}^{i-1} E_{k}}{\sum_{k=i_{e e}+1}^{i} L_{k}}-\alpha>P_{e e}, \forall i>i_{e e}
$$

Therefore, we can always find a time duration with $\hat{P}(t)=$ $\hat{P}^{\text {on }}>P_{e e}, t \in\left(\hat{t}^{\text {on }}, \hat{t}^{\text {on }}+\Delta \hat{t}^{\text {on }}\right) \subset\left(t_{i_{e e}}, T\right]$, and construct a new policy $\bar{P}(t)$ with $\bar{P}(t)=\bar{P}^{\text {on }}=\frac{\left(\hat{P}^{\text {on }}+\alpha\right) \Delta \hat{t}^{\text {on }}}{\Delta \hat{t}^{\text {on }}+\delta}-\alpha, t \in$ $\left(\hat{t}^{\mathrm{on}}, \hat{t}^{\mathrm{on}}+\Delta \hat{t}^{\mathrm{on}}\right) \cup\left(\hat{t}^{\mathrm{off}}, \hat{t}^{\mathrm{off}}+\delta\right)$. Note that we have chosen $\delta$ to be sufficiently small so that $\left(\hat{t}^{\text {off }}, \hat{t}^{\text {off }}+\delta\right) \subseteq\left(\hat{t}^{\text {off }}, \hat{t}^{\text {off }}+\right.$ $\left.\Delta \hat{t}^{\text {off }}\right)$ and $\bar{P}^{\text {on }}>P_{e e}$. The energy consumed by $\bar{P}(t)$ during $\left(\hat{t}^{\text {on }}, \hat{t}^{\text {on }}+\Delta \hat{t}^{\text {on }}\right) \cup\left(\hat{t}^{\text {off }}, \hat{t}^{\text {off }}+\delta\right)$ is $\hat{E}=\left(\bar{P}^{\text {on }}+\alpha\right)\left(\Delta \hat{t}^{\text {on }}+\delta\right)=$
$\left(\hat{P}^{\text {on }}+\alpha\right) \Delta \hat{t}^{\text {on }}$, which is same as the initial policy $\hat{P}(t)$. The throughput for the newly constructed policy $\bar{P}(t)$ and initial policy $\hat{P}(t)$ during $\left(\hat{t}^{\text {on }}, \hat{t}^{\text {on }}+\Delta \hat{t}^{\text {on }}\right) \cup\left(\hat{t}^{\text {off }}, \hat{t}^{\text {off }}+\delta\right)$ are

$$
B_{1}=R\left(\bar{P}^{\mathrm{on}}\right)\left(\Delta \hat{t}^{\mathrm{on}}+\delta\right)=R\left(\bar{P}^{\mathrm{on}}\right) \frac{\hat{E}}{\bar{P}^{\mathrm{on}}+\alpha}
$$

and

$$
B_{2}=R\left(\hat{P}^{\mathrm{on}}\right) \Delta \hat{t}^{\mathrm{on}}=R\left(\hat{P}^{\mathrm{on}}\right) \frac{\hat{E}}{\hat{P}^{\mathrm{on}}+\alpha}
$$

respectively. Since $\hat{P}^{\text {on }}>\bar{P}^{\text {on }}>P_{e e}$, and $\frac{R(x)}{x+\alpha}$ is monotonically decreasing as a function of $x$ when $x>P_{e e}$, we conclude that $B_{1}>B_{2}$. Therefore, the new policy achieves a higher throughput than the initial policy.

Moreover, we need to check that the new policy also satisfies the energy constraint as follows. If $\hat{t}^{\text {off }}>\hat{t}^{\text {on }}+\Delta \hat{t}^{\text {on }}$, or $\left(\hat{t}^{\text {on }}, \hat{t}^{\text {on }}+\Delta \hat{t}^{\text {on }}\right)$ and $\left(\hat{t}^{\text {off }}, \hat{t}^{\text {off }}+\delta\right)$ are in the same epoch, it is evident that the energy constraint is satisfied. If we cannot find an interval ( $\left.\hat{t}^{\text {off }}, \hat{t}^{\text {off }}+\Delta \hat{t}^{\text {off }}\right)$ latter than or in the same epoch as $\left(\hat{t}^{\text {on }}, \hat{t}^{\text {on }}+\Delta \hat{t}^{\text {on }}\right)$, i.e., all the allocated power prior to $\hat{t}^{\text {off }}$ is smaller than $P_{e e}$, in this case based on (46), there must be some energy left at time $\hat{t}^{\text {off }}$. Since we selected $\delta$ to be sufficiently small, we can still guarantee that the energy constraint is satisfied. Therefore, we prove that $l_{i}^{\text {on* } *}=L_{i}, i=i_{e e}+1, \ldots, N$.

After determining $l_{i}^{\text {on* }}=L_{i}, i=i_{e e}+1, \ldots, N$, we realize that Problem $\mathbb{P}_{2}$ for the last $N-i_{e e}$ epochs has the same structure as that studied in [12], and thus [12, Theorem 1] is applicable here. After some change of notation, we can prove that the solution in (15), (16) and (17) is optimal for $\mathbb{P}_{2}$ over the last $N-i_{e e}$ epochs.

Next, we show that the optimal solutions of $\mathbb{P}_{1}$ and $\mathbb{P}_{2}$ are optimal for (6) to complete the proof for Theorem 3.1 First, we can verify by following the similar contradiction proof in the above that any energy harvested during $\left[0, t_{i_{e e}}\right)$ should be used up in the first $i_{e e}$ epochs, i.e., $\sum_{j=1}^{i_{e e}}\left(P_{j}^{*}+\alpha\right) l_{j}^{\text {on* }}=$ $\sum_{j=0}^{i_{e e}-1} E_{j}$. Therefore, the energy constraint for $(6)$ is equivalent

$$
\begin{aligned}
& \sum_{j=1}^{i}\left(P_{j}+\alpha\right) l_{j}^{\text {on }} \leq \sum_{j=0}^{i-1} E_{j}, i=1, \ldots, i_{e e} \\
& \sum_{j=i_{e e}+1}^{i}\left(P_{j}+\alpha\right) l_{j}^{\text {on }} \leq \sum_{j=i_{e e}}^{i-1} E_{j}, i=i_{e e}+1, \ldots, N .
\end{aligned}
$$

Since the energy constraint is decoupled before and after $t_{i_{e e}}$, solving (6) is equivalent to optimizing $P_{i}$ and $l_{i}^{\text {on }}$ over $i=$ $1, \ldots, i_{e e}$ and $i=i_{e e}+1, \ldots, N$ separately. Thus, the optimal solutions for $\mathbb{P}_{1}$ and $\mathbb{P}_{2}$ are also optimal for (6). Theorem 3.1 is thus proved.

\section{APPENDIX D \\ PROOF OF PROPOSITION 4.1}

The first and third properties of $\bar{R}(P(t))$ can be directly verified by the first and third properties of $R(\mathbf{Q}(t))$, respectively. Thus, to complete the proof of Proposition 4.1, we only need to show the second property of $\bar{R}(P(t))$, i.e., it is a strictly 
concave function of $P(t)$. Similar to [18, Appendix B], we show the proof of this result as follows.

Since $\bar{R}(P(t))$ is obtained as the optimal value of (23), which is a convex optimization problem and satisfies the Slater's condition [17]. Thus, the duality gap for this problem is zero. As a result, $\bar{R}(P(t))$ can be equivalently obtained as the optimal value of the following min-max optimization problem:

$$
\begin{aligned}
\bar{R}(P) & =\min _{\mu \geq 0} \max _{Q_{k} \geq 0} R(\mathbf{Q})-\mu\left(\sum_{k=1}^{K} Q_{k}-P\right) \\
& =\min _{\mu \geq 0} R\left(\mathbf{Q}^{(\mu)}\right)-\mu \sum_{k=1}^{K} Q_{k}^{(\mu)}+\mu P \\
& =R\left(\mathbf{Q}^{\left(\mu^{(P)}\right)}\right)-\mu^{(P)} \sum_{k=1}^{K} Q_{k}^{\left(\mu^{(P)}\right)}+\mu^{(P)} P
\end{aligned}
$$

where we have removed $t$ for brevity, and in 49) $\mathbf{Q}^{(\mu)}=$ $\left[Q_{1}^{(\mu)}, \ldots, Q_{K}^{(\mu)}\right]$ is the optimal solution for the maximization problem with a given $\mu$, while in 50 $\mu^{(P)}$ is the optimal solution for the minimization problem with a given $P$. Since $R(\mathbf{Q}(t))$ is a strictly joint concave function, the optimal solutions in the above must be unique. Denote $\omega$ as any constant in $[0,1]$. Let $\mu^{\left(P_{1}\right)}, \mu^{\left(P_{2}\right)}$ and $\mu^{\left(P_{3}\right)}$ be the optimal $\mu$ for $\bar{R}\left(P_{1}\right), \bar{R}\left(P_{2}\right)$ and $\bar{R}\left(P_{3}\right)$ with $P_{3}=\omega P_{1}+(1-\omega) P_{2}$, respectively. For $j=1,2$, we have

$$
\begin{aligned}
\bar{R}\left(P_{j}\right) & =R\left(\mathbf{Q}^{\left(\mu^{\left(P_{j}\right)}\right)}\right)-\mu^{\left(P_{j}\right)} \sum_{k=1}^{K} Q_{k}^{\left(\mu^{\left(P_{j}\right)}\right)}+\mu^{\left(P_{j}\right)} P_{j} \\
& \leq R\left(\mathbf{Q}^{\left(\mu^{\left(P_{3}\right)}\right)}\right)-\mu^{\left(P_{3}\right)} \sum_{k=1}^{K} Q_{k}^{\left(\mu^{\left(P_{3}\right)}\right)}+\mu^{\left(P_{3}\right)} P_{j}
\end{aligned}
$$

where strict inequality holds for 52 if $P_{j} \neq P_{3}$ since the optimal $\mu^{\left(P_{j}\right)}, j=1,2$, are unique. Thus, we have

$$
\begin{aligned}
& \omega \bar{R}\left(P_{1}\right)+(1-\omega) \bar{R}\left(P_{2}\right) \\
\leq & R\left(\mathbf{Q}^{\left(\mu^{\left(P_{3}\right)}\right)}\right)-\mu^{\left(P_{3}\right)} \sum_{k=1}^{K} Q_{k}^{\left(\mu^{\left(P_{3}\right)}\right)}+\mu^{\left(P_{3}\right)} P_{3} \\
= & \bar{R}\left(P_{3}\right) \\
= & \bar{R}\left(\omega P_{1}+(1-\omega) P_{2}\right),
\end{aligned}
$$

where strict inequality holds for 54 if $\omega \in(0,1)$. Therefore, $\bar{R}(P(t))$ is a strictly concave function over $P(t) \geq 0$. The proof of Proposition 4.1 is thus completed.

\section{APPENDIX E \\ PROOF OF PROPOSITION 5.1}

We prove this proposition by considering the following two cases.

First, consider the case when $i_{e e}$ exists, i.e., $i_{e e} \in$ $\{1, \ldots, N\}$. In this case, setting $P_{e e}$ as the transmit power at time 0 is optimal according to Table \ Furthermore, we have $\frac{\sum_{k=0}^{i_{e e}, 1} E_{k}}{\sum_{k=1}^{i_{e} e e, 1} L_{k}}-\alpha<P_{e e}$ based on 111), and thus

$$
\min _{i=1, \ldots, N}\left(\frac{\sum_{k=0}^{i-1} E_{k}}{\sum_{k=1}^{i} L_{k}}-\alpha\right) \leq \frac{\sum_{k=0}^{i_{e e, 1}-1} E_{k}}{\sum_{k=1}^{i_{e e, 1}} L_{k}}-\alpha<P_{e e}
$$

Thus, 29) is equivalent to $P^{*}(0)=P_{e e}$, which is the optimal solution.

Next, consider the case when $i_{e e}$ does not exist, which implies that $\frac{\sum_{k=0}^{j-1} E_{k}}{\sum_{k=1}^{j} L_{k}}-\alpha>P_{e e}, \forall j=1, \ldots, N$. Thus, we have

$$
\min _{i=1, \ldots, N}\left(\frac{\sum_{k=0}^{i-1} E_{k}}{\sum_{k=1}^{i} L_{k}}-\alpha\right)>P_{e e}
$$

and (29) is equivalent to $P^{*}(0)=\min _{i=1, \ldots, N}\left(\frac{\sum_{k=0}^{i-1} E_{k}}{\sum_{k=1}^{i} L_{k}}-\alpha\right)$, which is the optimal solution according to Table \

From the above two cases, 29 coincides with the optimal solution for $P^{*}(0)$. Thus, Proposition 5.1 is proved.

\section{REFERENCES}

[1] T. Chen, Y. Yang, H. Zhang, H. Kim, and K. Horneman, "Network energy saving technologies for green wireless access networks," IEEE Wireless Commun., vol. 18, no. 5, pp. 30-38, Oct. 2011.

[2] C. Han et al., "Green radio: Radio techniques to enable energy-efficient wireless networks," IEEE Commun. Mag., vol. 49, no. 6, pp. 46-54, Jun. 2011.

[3] Z. Niu, Y. Wu, J. Gong, and Z. Yang, "Cell zooming for cost-efficient green cellular networks," IEEE Commun. Mag., vol. 48, no. 11, pp. 74-78, Nov. 2010.

[4] Z. Hasan, H. Boostanimehr, and V. Bhargava, "Green cellular networks: A survey, some research issues and challenges," IEEE Commun. Surveys \& Tutorials, vol. 13, no. 4, pp. 524-540, 2011.

[5] Y. Chen, S. Zhang, S. Xu, and G. Y. Li, "Fundamental trade-offs on green wireless networks," IEEE Commun. Mag., vol. 49, no. 6, pp. 30-37, Jun. 2011.

[6] E. Uysal-Biyikoglu, B. Prabhakar, and A. Gamal, "Energy-efficient packet transmission over a wireless link," IEEE/ACM Trans. Network., vol. 10, no. 4, pp. 487-499, Aug. 2002.

[7] G. W. Miao, N. Himayat, and G. Y. Li, "Energy-efficient link adaptation in frequency-selective channels," IEEE Trans. Commun., vol. 58, no. 2 , pp. 545-554, Feb. 2010.

[8] C. Isheden, Z. Chong, E. Jorswieck, and G. Fettweis, "Framework for link-level energy efficiency optimization with informed transmitter," IEEE Trans. Wireless Commun., vol. 11, no. 8, pp. 2946-2957, Aug. 2012.

[9] O. Blume, H. Eckhardt, S. Klein, E. Kuehn, and W. M. Wajda, "Energy savings in mobile networks based on adaptation to traffic statistics," Bell Labs Tec. J., vol. 15, no. 2, pp. 77-94, Sep. 2010.

[10] H. Kim and G. Veciana, "Leveraging dynamic spare capacity in wireless system to conserve mobile terminals energy," IEEE/ACM Trans. Network. vol. 18 , no. 3, pp. 802-815, Jun. 2010.

[11] A. Kansal, J. Hsu, S. Zahedi, and M. B. Srivastava, "Power management in energy harvesting sensor networks," ACM Trans. Embed. Comput. Syst., vol. 6, no. 4, Sep. 2007.

[12] J. Yang and S. Ulukus, "Optimal packet scheduling in an energy harvesting communication system," IEEE Trans. Commun., vol. 60, no. 1, pp. 220-230, Jan. 2012.

[13] C. K. Ho and R. Zhang, "Optimal energy allocation for wireless communications with energy harvesting constraints," IEEE Trans. Sig. Process., vol. 60, no. 9, pp. 4808-4818, Sep. 2012.

[14] K. Tutuncuoglu and A. Yener, "Optimum transmission policies for battery limited energy harvesting nodes," IEEE Trans. Wireless Commun., vol. 99, no. 2, pp. 1-10, Feb. 2012.

[15] Q. Bai, J. Li, and J. A. Nossek, "Throughput maximizing transmission strategy of energy harvesting nodes," in Proc. IWCLD, Rennes, France, Nov. 2011.

[16] A. Goldsmith, Wireless Communications. Cambridge, U.K.: Cambridge Univ. Press, 2005.

[17] S. Boyd and L. Vandenberghe, Convex Optimization, Cambridge University Press, 2004.

[18] R. Zhang, F. Gao, and Y.-C. Liang, "Cognitive beamforming made practical: Effective interference channel and learning-throughput tradeoff," IEEE Trans. Commun., vol. 58, no. 2, pp. 706-718, Feb. 2010.

[19] B. Devillers and D. Gunduz, "A general framework for the optimization of energy harvesting communication systems with battery imperfections," Journal of Commun. and Netw., vol. 14, no. 2, pp. 130-139, Apr. 2012. 